\title{
P-139
}

\section{IR-Metabolomics Aprroach in Detecting the Possible Changes of Andrographis Paniculata Metabolites Due to Different Harvesting Ages and Times}

\author{
Nur A’thifah Yusof ${ }^{1}$, Azizul Isha ${ }^{1}$ and Intan Safinar Ismail ${ }^{1,2,{ }^{*}}$ \\ ${ }^{1}$ Laboratory of Natural Products, Institute of Bioscience, Universiti Putra Malaysia, 43000 Serdang, Malaysia; \\ ${ }^{2}$ Department of Chemistry, Faculty of Science, Universiti Putra Malaysia, 43000 Serdang, Malaysia; \\ E-mail:intan@science.upm.edu.my
}

This study was designed to investigate the effect of different harvesting ages and times on the changes of the metabolite functional groups in three germplasm accessions of Malaysia Andrographis paniculata (Burn. F.) Nees, viz 11265, 11341, 11248. The study was focused on the plant leaf as this part constitutes a high amount of interesting bioactive compounds which among others are the diterpenes lactones. The plants were harvested at three different ages of 120,150 and 180 days after transplanting (DAT). Each harvest was carried out at 2 different times, morning (around $7.30 \mathrm{am}$ ) and evening (around 3.00 pm). The leaves methanol extract of each different age and harvest time was analyzed by using Fourier Transform Infrared (FTIR). The variations in the metabolites observed in the FT-IR spectroscopic data of the sample extracts paved the way in identifying the differences due to the harvesting time and age. By using the multivariate data analysis (PCA and PLS-DA) for the different harvesting time samples, the differences of the functional groups were observed between the range $1630-1600 \mathrm{~cm}^{-1}$ which represent $\mathrm{C}=\mathrm{C}$ functionality and the band pattern between within the $1000-1400 \mathrm{~cm}^{-1}$. For the harvesting ages, the work is in progress.

Keywords: Andrographis paniculata, FT-IR, Functional groups, PLS-DA. 